RUNNING HEAD: attention training and reappraisal

\title{
Attention training through gaze-contingent feedback: \\ Effects on reappraisal and negative emotions
}

\author{
Alvaro Sanchez, Jonas Everaert, and Ernst H. W. Koster \\ Ghent University, Belgium
}

Key words: Selective attention; attentional control; reappraisal; negative emotions; attention bias modification

Abstract: 224 words

Article body (Introductory and discussion materials, and acknowledgments): 1,966 words References: 40

Number of tables: 4

Number of figures: 7

Supplemental material: 1

\author{
* Corresponding author: \\ Alvaro Sanchez \\ Ghent University \\ Department of Experimental Clinical and Health Psychology \\ Henri Dunantlaan 2 \\ B-9000 Ghent \\ Belgium \\ Tel: +0032092649105 \\ Fax: +0032092646489 \\ E-mail: alvaro.sanchezlopez@ugent.be
}




\begin{abstract}
Reappraisal is central to emotion regulation but its mechanisms are unclear. This study tested the theoretical prediction that emotional attention bias is linked to reappraisal of negative emotioneliciting stimuli and subsequent emotional responding using a novel attentional control training. Thirty-six undergraduates were randomly assigned to either the control or the attention training condition and were provided with different task instructions while they performed an interpretation task. Whereas control participants freely created interpretations, participants in the training condition were instructed to allocate attention toward positive words to efficiently create positive interpretations (i.e., recruiting attentional control) while they were provided with gazecontingent feedback on their viewing behavior. Transfer to attention bias and reappraisal success was evaluated using a dot-probe task and an emotion regulation task which were administered before and after the training. The training condition was effective at increasing attentional control and resulted in beneficial effects on the transfer tasks. Analyses supported a serial indirect effect with larger attentional control acquisition in the training condition leading to negative attention bias reduction, in turn predicting greater reappraisal success which reduced negative emotions. Our results indicate that attentional mechanisms influence the use of reappraisal strategies and its impact on negative emotions. The novel attention training highlights the importance of tailored feedback to train attentional control. The findings provide an important step toward personalized delivery of attention training.
\end{abstract}




\section{Introduction}

Reappraising the meaning of an emotion-eliciting event to decrease its negative impact is a powerful regulatory process integral to healthy as well as distorted emotional functioning (Gross, 2014). In nonclinical samples, reappraisal is effective at increasing positive and decreasing negative emotions (Augustine \& Hemenover, 2009; Webb, Miles, \& Sheeran, 2012) and has been associated with better interpersonal functioning (Gross \& John, 2003) as well as enhanced stress recovery (Jamieson, Nock, \& Mendes, 2012). Failures to reappraise have been reported in depressed individuals (Aldao, Nolen-Hoeksema, \& Schweizer, 2010; Johnstone, van Reekum, Urry, Kalin, \& Davidson, 2007). Models of reappraisal suggest that attentional mechanisms determine reappraisal success (Joormann \& D’Avanzato, 2010; Sheppes, Suri, \& Gross, 2015) and that unsuccessful reappraisal in depression can be related to attentional impairments and biases in processing emotional material (De Raedt \& Koster, 2010).

An upsurge of research examining the role of attention allocation in reappraisal of emotional material has yielded equivocal findings. Some investigations have found that participants spent less time looking at emotional regions of negative material when downregulating negative emotions (Manera, Samson, Pehrs, Lee, \& Gross, 2014; van Reekum et al., 2007), and that such shorter viewing times mediate the effect of reappraisal on the negative emotions experienced (Manera et al., 2014). Other studies, however, have reported the reverse, namely longer viewing times for emotional content during reappraisal (Bebko, Franconeri, Ochsner, \& Chiao, 2011) and that constraining participants' gaze toward neutral vs. negative regions of emotional pictures does not alter reappraisal success (Bebko, Franconeri, Ochsner, \& Chiao, 2014; Urry, 2010). One explanation for the mixed findings could be that effective 
reappraisal can be achieved in multiple ways, differentially recruiting attentional resources (Morris, Leclerc, \& Kensinger, 2014).

Thus far, research has been limitedly successful at characterizing the role of emotional attention (bias) in reappraisal, especially because most studies are cross-sectional and preclude claims regarding causality (but see Bebko et al., 2014). How attentional mechanisms directly contribute to reappraisal has not been investigated thoroughly. One common technique to assess and manipulate attentional allocation draws from the emotional dot-probe paradigm (MacLeod, Rutherford, Campbell, Ebsworthy, \& Holker, 2002). Here, participants learn to allocate attention toward or away from threats via experimental contingencies between the presentation of stimuli (either threatening or neutral) and a to-be-detected target. Although initial reports on the effectiveness of dot-probe training were promising (Hakamata et al., 2010), recent meta-analyses indicate that the training has only modest effects in modifying biases in attentional allocation (Beard, Sawyer, \& Hofmann, 2012; Mogoașe, David, \& Koster, 2014). Factors limiting the effectiveness of training are the limited benefit of picking up the contingency between a particular emotional picture and the subsequently presented target in a dot-probe training task as well as the lack of individualized feedback on trainees' performance during attention training. Initial empirical evidence suggests that providing online feedback during training increases awareness of emotional biases in attention allocation, which can in turn increase regulatory control (Bernstein \& Zvielli, 2014; Schnyer et al., 2015). Novel attentional control training techniques could improve training effects via tailored feedback to take full advantage of a trainee's ability to implement self-regulatory control in redirecting attention (i.e., attentional control). 
The present study investigated the direct influence of emotional attention (bias) in reappraisal using a novel attentional control training methodology implementing gaze-contingent feedback. For this purpose, we modified a recently designed method to investigate attentional bias during interpretation (Everaert, Duyck, \& Koster, 2014; Sanchez, Everaert, De Putter, Mueller, \& Koster, 2015) into an attentional control training task. In the basic task design, participants unscrambled emotional sentences (e.g., "future dismal very my bright looks") using five of the six words into either a positive or a negative sentence, while their gaze behavior was recorded. In the training variant, participants were instructed to guide attention allocation toward positive words to create positive sentences and were provided with gaze-contingent feedback on their viewing times to positive vs. negative words of the emotional scrambled sentences. Therefore, participants in the attentional control condition were explicitly instructed to implement top-down regulation of their attention patterns (i.e., increase visual fixation to positive over negative words), and received gaze-contingent feedback in order to maximize such regulation of attention according to the explicitly instructed pattern. To assess the effectiveness of the attentional control training in modifying attentional bias and reappraisal, we administered a dot-probe task and an emotion regulation task before and after the modification procedure. We hypothesized that gaze-contingent feedback in the training condition would increase attentional control (i.e., significant changes in attention bias in the training group from a baseline to a modification phase during the training), transferring to attentional bias on the dot-probe task, and affecting reappraisal success in the emotion regulation task. 


\section{Method}

\section{Participants}

To obtain large variability in negative attentional bias, participants with minimal and severe depressive symptoms were sampled from the Ghent University research participant pool based on a prescreening measure (Mood and Anxiety Symptom Questionnaire; Watson, Clark, Weber, Assenheimer, Strauss, \& McCormick, 1995). At testing, 40 participants (33 women; 1829 years) reported a broad range of depressive symptom severity levels (range: $0-42, M=13.28$, $S D=9.15$ ) on the Beck Depression Inventory-II (Beck, Steer, \& Brown, 1996; Van der Does, 2002). Sample size in the study was determined based on previous research reporting effects of attentional control training procedures in attention bias modification (Bernstein \& Zvielli, 2014). Participants were paid 15 euro. The institutional review board approved the study protocol.

\section{Design Overview}

Figure 1 depicts the sequence of tasks. All participants completed a baseline phase followed by either a training or control modification phase that was determined by random assignment. Before the baseline and after the modification phase, participants completed the emotion regulation and dot-probe tasks. An experimental session lasted approximately 85 min.

\section{Training Procedure}

Baseline phase. Eye movements were monitored via eye tracking while participants completed an interpretation task, the Scrambled Sentences Test (Everaert, Duyck, et al., 2014). On each trial (Figure 2), a neutral (e.g., "the I theatre visit cinema often”) or emotional (e.g., "am winner born loser a I') scrambled sentence was displayed following fixation (left-aligned to elicit left-to-right reading). While the item was on-screen, participants were instructed to 
unscramble the sentence to form a grammatically correct and meaningful statement using five of the six words as quickly as possible and within a time limit of $8000 \mathrm{~ms}$ (e.g., "I am a born winner"). Upon completion, they pressed a button to continue and report their solution using the numbers linked to the words of a scrambled sentence.

After a 3-trial practice phase with neutral scrambled sentences, 12 emotional scrambled sentences were presented in random order. Participants then completed 6 filler neutral scrambled sentences before starting the modification phase.

Modification phase. In both the training and control condition of the modification phase, participants completed 8 blocks of 6 randomly presented emotional scrambled sentences. Again, eye movements were registered while participants unscrambled the sentences. While the task in the control condition was identical to the baseline task phase, several manipulations were made in the training condition (Figure 3). First, participants were instructed to unscramble all sentences into positive self-statements (Sanchez et al., 2015) and to focus attention on positive words, as this would help to identify and form positive meanings more efficiently. Second, participants received online feedback about their attentional deployment while unscrambling the sentences. A red or green square respectively framed the negative or positive target each time the eye-tracker detected a fixation. This online feedback aimed to help participants to quickly disengage from negative information and maintain attention to positive information. Finally, after each training block, participants received feedback comparing their gaze behavior during the last block (e.g., "You looked 54\% of the time at the positive word") with gaze behavior during the baseline phase (e.g., "You looked $42 \%$ of the time at the positive word"). This procedure intends to increase awareness of the progress made in the training condition compared with baseline. 
Stimuli. Sixty-nine scrambled sentences (60 emotional, 9 neutral sentences) were drawn from a prior study (Everaert, Duyck, et al., 2014). All scrambled sentences were self-referent and 6 words long. Negative and positive target words in each emotional sentence (e.g., "winner" and "loser" in "am winner born loser a I") were matched between valence categories on word length, word class, and word frequency (Duyck, Desmet, Verbeke, \& Brysbaert, 2004), all $F$-s $<1$. Word position within each scrambled sentence was randomized with the constraint that target words occurred neither next to each other nor as the first or last word within a scrambled sentence. Positive and negative target word order in emotional sentences was counterbalanced. The same criteria were applied to target words in the neutral sentences.

Dependent variables. The eye-tracker recorded online the total fixation times (sum of durations across fixations) on positive and negative target words in emotional scrambled sentences during both the baseline and modification phase. An index of attention bias in processing positive vs. negative material was computed by dividing the total fixation time on positive words by the total fixation time on emotional (positive and negative) words (Everaert, Duyck, et al., 2014; Sanchez et al., 2015) separately for each training phase (i.e., baseline phase vs. modification phase). These attention bias indices served to test the hypothesis that participants would implement attentional regulation (i.e., attentional control acquisition) in the training condition, by showing significant increases in attention bias to positive over negative material from the baseline to the modification phase.

Similarly, an index of interpretation bias was computed by dividing the number of positively unscrambled sentences by the total number correctly completed emotional (positive and negative) sentences (Everaert, Duyck, et al., 2014; Sanchez et al., 2015) separately for the 
baseline and modification phases, which served to test the change in interpretation bias from the baseline to the modification phase.

\section{Transfer of training}

\section{Attention bias}

An emotional dot-probe task (MacLeod, Mathews, \& Tata, 1986) indexed transfer of the attentional control training to attention bias (Figure 4). After central fixation, each trial simultaneously presented two words (positive-negative or neutral-neutral pairs) for $1000 \mathrm{~ms}$ at either side of fixation (above vs. below fixation). After offset, a probe ("X") appeared with equal probability at the location of one of the stimuli. Participants were instructed to locate the probe as quickly and accurately as possible by pressing the corresponding buttons.

Forty-eight positive-negative and 24 neutral-neutral word pairs were selected. Positive, negative, and neutral words were matched on word length, word class, and word frequency (Duyck et al., 2004), all $F$-s $<1$. The total set of 288 trials ( 72 word pairs $\times 2$ word locations $\times 2$ probe locations) was divided to create two dot-probe versions. Each version contained 144 trials (96 positive-negative trials, 48 neutral-neutral trials) with word and probe location counterbalanced. The two versions served as pre- and post-training procedure measures of attention bias. Administration of the versions was counterbalanced across participants.

Data from the dot-probe tasks were trimmed to minimize the influence of outliers. Errors and RTs $<150 \mathrm{~ms}$ and $>1500 \mathrm{~ms}$ were removed and then RTs falling more than 3 SDs from each participant's mean RT were excluded (Everaert, Mogoase, David, \& Koster, 2014). Analyses were conducted on $98 \%$ of the data. An index of negative attention bias was calculated for pre- and post-training task versions. RTs on trials with probes replacing negative words (i.e., congruent trials) were subtracted from RTs on trials with probes replacing positive words (i.e. 
incongruent trials). Higher scores indicate a stronger attentional bias for negative words (MacLeod et al., 1986).

\section{Emotion Regulation}

An emotion regulation task (Figure 5) assessed transfer to reappraisal (Vanderhasselt, Kühn, \& De Raedt, 2013). On each trial, a negative picture was presented and, after $2000 \mathrm{ms,}$ participants rated their negative emotional experience on a 10 -point scale $(0$ - 'not at all' to $9-$ 'very much'). A cue subsequently prompted them to appraise or reappraise the picture's meaning. When instructed to appraise, participants were asked to look at the picture and freely experience the elicited feelings. When instructed to reappraise, participants were asked reinterpret the picture's meaning in a less negative way by changing the emotions, actions, and outcomes of individuals depicted in the picture (Ochsner, Bunge, Gross, \& Gabrieli, 2002). After $10 \mathrm{~s}$, participants' negative emotional experiences were reassessed using the same 9-point rating scale. When instructed to reappraise, participants also provided a description of how they reappraised the picture.

Stimuli and task versions. Thirty-two negative IAPS pictures (Lang, Bradley, \& Cuthbert, 2008) depicting depression-relevant themes (e.g., crying people, loneliness) were selected based on arousal $(M<4$, range 4.30-7.93) and valence ratings $(M<4$, range 1.37-3.72). Two sets of 16 pictures were created that differed neither on valence nor on arousal, all $p$-s $>.05$. One set was presented before and the other after the training procedure, counterbalanced across participants. In each task version, half of the pictures were appraised and the other half reappraised. Pictures and regulatory instructions were randomly presented with the constraint that maximum 2 pictures with the same regulatory instruction occurred consecutively. 
Dependent variables. First, reappraisal scores were computed using narrative descriptions provided by participants. Two blind raters evaluated whether participants were successful at generating reappraisals of negative scenes using a 5-point scale (0-No Description, 1-Not at all, 2-A little, 3-Good, 4-Very good). An intra-class correlation of .90 ( $p=.001)$ indicated high inter-rater agreement. Reappraisal scores were computed by averaging the blind raters' scores separately for the pre- and post-training emotion regulation tasks. Higher scores indicate better reappraisal. Second, negative emotion scores were computed by averaging the emotion ratings indicating the degree of negative emotions after viewing pictures, for the appraisal and reappraisal trials.

\section{Eye-tracker}

A Tobii TX300 eye-tracker recorded gaze behavior during the dot-probe tasks and the training procedure, with eye-gaze coordinates sampling at $300 \mathrm{~Hz}$. Participants were seated approximately $60 \mathrm{~cm}$ from the eye tracker. Visual fixations were considered when longer than $100 \mathrm{~ms}$. Stimulus presentation and eye movement recording were controlled by E-prime Professional software. E-prime extensions (TET and Clearview PackageCalls) converted eye movement signals to visual fixation data, and computed and presented fixation time scores in the training condition.

\section{Results}

\section{Sample Characteristics}

Four participants were excluded from the analyses due to problems in the detection of gaze position, low quality of eye-tracking recordings (valid samples $<75 \%$ ), or lack of fluency in Dutch. The final sample size was 36 individuals (30 women; $18-29$ years). The control and 
training condition did not significantly differ in age, $t(33)=0.22, p=.83$, gender ratio, $\chi^{2}(1)=0.17$, $p=.68$, nor depressive symptoms, $t(33)=-1.42, p=.16$. Table 1 presents descriptives on all study variables.

\section{Training Effectiveness}

A series of 2 (Condition: Training, Control) x 2 (Phase: Baseline, Modification) mixeddesign ANOVAs were conducted to examine effects of the attentional control training on changes in interpretation bias and attention bias across the training procedure (i.e., from the baseline to the modification phase). The first ANOVA employed the measures of interpretation bias at each phase as dependent variable, whereas the second ANOVA employed the measures of attention bias at each phase as dependent variables (see Table 1). Regarding interpretation bias change, analyses revealed a significant main effect of Phase, $F(1,34)=18.76, p=.001, \eta^{2}=.36$, qualified by a Condition $\times$ Phase interaction, $F(1,34)=18.20, p=.001, \eta^{2}=.35$. Follow-up Bonferroni-corrected comparisons showed no differences between conditions at the Baseline phase, $F(1,34)=0.16, p=.69, \eta^{2}=.01$. At the Modification phase the training condition reported a higher positive interpretation bias compared to the control condition, $F(1,34)=37.38, p=.001$, $\eta^{2}=.52$. As expected, a significant increase in positive interpretations was found from Baseline to Modification in the training, $F(1,34)=36.96, p=.001, \eta^{2}=.52$, but not in the control condition, $F(1,34)=0.01, p=.96, \eta^{2}=.01$.

Regarding attention bias change, analyses revealed a main effect of Phase, $F(1,34)=15.74, p=.001, \eta^{2}=.32$, qualified by a Condition $\times$ Phase interaction, $F(1,34)=31.14$, $p=.001, \eta^{2}=.48$. Follow-up tests showed no differences between conditions in attention bias at the Baseline phase, $F(1,34)=1.62, p=.21, \eta^{2}=.04$, but significant differences at the Modification phase, $F(1,34)=38.76, p=.001, \eta^{2}=.53$, with participants in the training condition showing a 
larger positive attention bias (i.e. more time attending to positive over negative words in the modification phase in comparison to participants in the control condition). As expected, there was a significant increase in attention bias from Baseline to Modification phase in the training, $F(1,34)=45.58, p=.001, \eta^{2}=.57$, but not in the control condition, $F(1,34)=1.30, p=.26, \eta^{2}=.04$ (i.e., significant increase in the time attending to positive over negative material from the baseline to the modification phase in the training condition). Further comparisons of each attention bias score at each phase of the procedure against a value of 0.5 (i.e., indicative of absence of bias) showed no biases during the baseline phase in either the training or the control condition, $p=.51$ and $p=.99$, respectively. In contrast, whereas the control group continued showing absence of bias during the modification phase, $p=.61$, the increase in the trained pattern in the training group was qualified by a bias to fixate more time in positive than in negative material during the modification phase, $p=.001$. Overall, these results suggest that the attentional control training was effective in increasing attention toward positive relative to negative information by implementing attentional control on the trained pattern. ${ }^{1}$

\section{Transfer of Training}

Given that prior research has revealed marked individual differences in the malleability of attention bias through training (Clarke, Chen, \& Guastella, 2012; Clarke, Macleod, \& Shirazee, 2008; Everaert et al., 2014) and that there also was substantial variability in attention bias scores both in the attentional control training and in the dot-probe task in the current study (see Table

\footnotetext{
${ }^{1}$ Further analyses were conducted to establish whether the manipulations introduced in the training condition during the modification phase would also lead to a faster performance on the task (i.e., faster times to unscramble sentences at the modification phase in comparison to the baseline phase in the training condition). A 2 (Condition: Training, Control) x 2 (Phase: Baseline, Modification) mixed-design ANOVA with the mean time to unscramble the sentences as dependent variable showed a marginally significant Condition by Phase interaction, $F(1,32)=3.35, p=.07, \eta^{2}=.09$. Bonferroni-corrected comparisons showed a significant reduction in the time to perform the task from the Baseline to Modification Phase in the training condition, Baseline: $4728 \mathrm{~ms}(\mathrm{SD}=1107)$, Modification: $4111 \mathrm{~ms}(\mathrm{SD}=1089)$, $p=.001$, whereas a similar trend did not reach significance in the control condition, Baseline: $4571 \mathrm{~ms}(\mathrm{SD}=1190)$, Modification: $4335 \mathrm{~ms}$ ( $\mathrm{SD}=913), p=.11$. These results suggest that the training group increased their efficiency to perform the task as the result of the manipulation procedures introduced during the modification phase.
} 
1), we focused the statistical analyses on the individual differences when evaluating transfer of the attentional control training to the dot-probe and emotion regulation tasks.

Individual differences were analyzed via residualized change scores, constructed using simple linear regression models (Segal et al., 2006). First, attentional control acquisition in the training procedure was indexed by computing change scores in attention bias from the baseline to the modification phase. Attention bias scores during the baseline phase were entered in a simple regression model as predictor of attention bias scores during the modification phase. The resulting standardized residuals served as a measure of attentional control acquisition. Second, in a similar way, changes in attention bias in the dot-probe task, and in reappraisal and negative emotions after reappraisal in the emotion regulation task were indexed by computing change scores from the pre- to the post-training for each of these measures separately. Each simple regression model regressed the post-training score on the pre-training score (i.e., time 1 score predict time 2 score, repeated for: attention bias in the dot-probe task, reappraisal and negative emotions after reappraisal in the emotion regulation task). The resulting standardized residuals of each regression model served as change scores. Using standardized residuals is a reliable method to control for variability among differences in the baseline scores (Segal et al., 2006). Table 2 presents correlations among the change scores.

Serial mediation models were used to examine effects of the modification condition (i.e. training vs. control) on changes in dot-probe attention bias, reappraisal, and negative emotions, via attentional control acquisition. After testing the significance of the total and direct effects, the significance of the indirect effect for each model was tested using a 5000 samples bias-corrected bootstrapping procedure (Preacher \& Hayes, 2008). Bootstrapping is a nonparametric approach to estimate the magnitude and significance of indirect effects and is recommended for use with 
small samples (Preacher \& Hayes, 2008). The estimated 95\% bootstrap confidence intervals should not contain 0 to be significant (Preacher \& Hayes, 2008). The effect size of each indirect effect model was derived by computing partially standardized indirect effects. This approach is indicated when the predictor variable is a dichotomous variable in which the two groups differ by one unit ( i.e., 0 - Control condition, 1 - Modification condition; see Preacher \& Kelley, 2011). Partially standardized effect sizes can be interpreted as the number of standard deviations in the outcome that the groups differed on average as result of the indirect mechanisms tested. Further results from single-step multiple regression analyses testing each mediational model are provided as supplemental material.

\section{Transfer to the dot-probe task}

A first mediation model examined the effect of modification condition (training vs. control) on dot-probe attention bias change via attentional control acquisition. Neither the total effect, $\mathrm{c}=-.29(S E=.34), t=-0.85, p=.40,95 \%-\mathrm{CI}:[-.9834, .4033]$, nor the direct effect, $\mathrm{c}^{\prime}=.66$ $(S E=.42), t=-1.57, p=.13,95 \%$-CI: [-.1952, 1.5203], were significant. The indirect effect was negative (coefficient=-.95, SE=.63) and statistically different from zero, 95\%-CI: [-2.4692, .0092], supporting the model. Partially standardized indirect effect of the model was -.93 $(S E=.52 ; 95 \%-C I:[-2.0829,-.0050])$, showing that the training modification condition was associated with decreases of .93 standard deviations in negative attention bias on the dot-probe via its effect on attentional control acquisition (Figure 6).

\section{Transfer to emotion regulation}

Effects on reappraisal. We tested a serial mediation model in which the modification condition predicts attentional control acquisition which in turn predicts attention bias change in the dot-probe and this predicts reappraisal change (outcome variable). The total effect, c=.06 
$(S E=.33), t=0.18, p=.86,95 \%$-CI: [-.6092, .7283], and direct effect, $\mathrm{c}^{\prime}=.40(S E=.44), t=0.90$, $p=.37,95 \%$-CI: $[-.4965,1.2907]$, were not significant. The indirect effect was positive (coefficient=.47, $S E=.41$ ) and statistically different from zero, 95\%-CI: [.0061, 1.6238]. Importantly, neither of the alternative models, where mediators were removed one-by-one (i.e., becoming covariates and, therefore, controlling for their influence on the other predictors), were significant (Table 3). Therefore, the only statistically supported indirect effect path was the one hypothesized. Partially standardized indirect effect of the supported model was .49 (SE=.41; 95\%-CI: $[.0038,1.5871])$. Thus, the training modification condition was indirectly associated with 0.49 standard deviations of reappraisal improvement via its effect on attention control acquisition, which in turn predicted attention bias change, which was associated with reappraisal change (Figure 7).

Effects on negative emotion. A final serial mediation model was tested adding negative emotional state after reappraisal to the previously validated indirect effect model: modification condition $\rightarrow$ attentional control acquisition $\rightarrow$ attention bias change $\rightarrow$ reappraisal change $\rightarrow$ negative emotional state after reappraisal (Figure 7). The total effect, $\mathrm{c}=.18(S E=.29), t=0.62$, $p=.54,95 \%$-CI: [-.4198, .7911], and direct effect, $\mathrm{c}^{\prime}=.39(S E=.40), t=0.97, p=.34,95 \%$-CI: [$.4307,1.2111]$, were not significant. The indirect effect was negative (coefficient $=-.22, S E=.22$ ) and statistically different from zero, 95\%-CI: [.-9512, -.0045], whereas neither of the alternative models were significant (Table 4). Therefore, the only statistically supported indirect effect path was the one hypothesized. The partially standardized indirect effect of the supported model was $.25(S E=.26 ; 95 \%-C I:[-1.0763,-.0034])$. Therefore, the training modification condition indirectly led to decreases by 0.25 standard deviations in negative emotion after reappraisal (i.e., better emotion regulation), via its influence in attention control acquisition, the influence of 
attentional control acquisition on attention bias change in the dot-probe, the influence of attention bias change on reappraisal change, and the influence of reappraisal change on negative emotion change.

\section{Discussion}

This study tested whether alteration of attention bias via a novel attentional control training task with gaze-contingent feedback would influence attention bias and reappraisal success assessed by transfer tasks. The results indicate that the training vs. control condition had an indirect effect on negative emotion repair: attentional control training led to attentional control acquisition during the training procedure, which predicted attention bias change in the dot-probe task, which in turn was associated with reappraisal change, which regulated negative emotion change. These transfer effects of attentional control training were specifically observed for individuals in the training and not in the control condition. The large individual differences in attentional control (acquisition) during the training indicated that the training was particularly effective for a subset of the trained individuals. The results support the proposal that attentional control training can modify attention bias, which in turn influences the use of cognitive reappraisal to decrease negative emotions.

Theoretical models of emotion regulation hypothesize that attentional mechanisms are causally related to emotion regulation strategies, including reappraisal and its impact on negative emotions (De Raedt \& Koster, 2010; Joormann \& D’Avanzato, 2010; Sheppes et al., 2015). Consistent with the theoretical predictions and prior research suggesting that down-regulating negative emotions is guided by less attention toward negative information (Manera et al., 2014; van Reekum et al., 2007), the present study showed that modifying attention bias via attentional 
control training influences reappraisal and negative emotionality. However, the present findings are in contrast with studies that have reported longer viewing times for negative content during reappraisal (Bebko et al., 2011) and no effects of attention manipulations on reappraisal success (Bebko et al., 2014; Urry, 2010). While there could be multiple routes to effective reappraisal (Morris et al., 2014), another explanation for this inconsistency is that these studies have only targeted overt attentional processes (i.e., fixating gaze position to a certain region of a negative picture) and do not control covert attentional shifts which may also explain reappraisal success. In the present study, both overt (i.e., eye movement indices) and covert (i.e., RTs on the dotprobe task) attentional shifts were indexed and related to reappraisal success. The established relation between attention and emotion reappraisal points to the importance of considering attentional mechanisms in understanding (and treating disordered) emotional wellbeing.

The novel training paradigm applied in this study may provide a promising tool to improve emotion regulation difficulties in remitted and/or clinically depressed individuals (Aldao et al., 2010; Johnstone et al., 2007). Interestingly, previous research (Sanchez et al., 2015) has shown that ability-related processes recruiting attentional control act as an intervening variable in the relation between depressive symptom levels and interpretation bias. Therefore, procedures increasing attentional control may help to reduce emotional dysregulation related to depression by affecting attentional mechanisms involved in successful reappraisal. Here, two aspects of the developed training procedure seem to be critical towards explaining its beneficial effects. First, different from the presentation of words or pictures in a standard dot-probe training, the present training procedure provided trainees with specific contexts (i.e., the content of the scrambled sentences), instructions, and feedback to help them in considering positive meanings in a self-referent manner. Second, the current training procedure provided 
individualized feedback on trainees' attention allocation performance during the training in order to maximize the regulation of attention according to an explicitly instructed pattern (i.e., intentionally (re)direct attention to positive information to form positive self-referent meanings). Both individualized feedback procedures involved the use of voluntary top-down strategies according to the instruction (online feedback: intentionally inhibit attention from negative words when they are fixated and maximize intentional visual search of positive words; feedback on performance between blocks: increase awareness on emotional biases to increase regulatory control in redirecting attention in subsequent trials; Bernstein \& Zvielli, 2014; Schnyer et al., 2015). Additionally, online gaze-contingent feedback may also tap into stimulus-driven bottomup factors that have been found to be relevant for the modification of emotional biases in attention (see Price, Greven, Siegle, Koster \& De Raedt, 2016). Further studies will require disentangling the specific effects of each of the feedback procedures comprising this new approach. It is noteworthy that the training and control condition differed on a number of elements, with the training including not only (1) different types of feedback, but also (2) the instruction to form positive sentences. One might wonder which components are most important in training attention. Noteworthy, in a previous study we have used a version of the SST where we instructed to form positive sentences without providing gaze-contingent feedback (in relation to a different research question; Sanchez et al., 2015). We found that attention did not change substantially in the SST where individuals were only instructed to form positive sentences, suggesting that feedback is a crucial element. Further studies should test the contribution of each of the feedback procedures in comparison to a condition only instructing to form positive scenes (without receiving feedback), helping to disentangle the specific adding of each feedback procedure to the explicit instruction. 
Broadly, this training is an important step towards more personalized and advanced cognitive training where biological indices directly provide feedback about performance. Future research will require extending the present findings by testing the transfer effects of this intervention to other sources of emotional information (e.g., effects in attention bias for emotional faces and scenes) using different methodologies to target covert and overt processes sub-serving attention bias as well as different indices of emotional functioning (i.e., self-reported subjective mood and objective physiological indicators of emotional functioning).

Some limitations should be acknowledged. First, the study was conducted in a nonclinical sample, which may limit generalizability of the findings to clinical samples. Given that attention biases observed in nonclinical samples of individuals with varying depression levels often differ from clinical samples in terms of degree rather than type (Baert, De Raedt, \& Koster, 2010; Beck \& Haigh, 2014), it can be expected that clinically depressed individuals experiencing profound attentional control and emotion regulation impairments (De Raedt \& Koster, 2010; Joormann \& D’Avanzato, 2010) may benefit more from the attentional control training tested in this study. Future research, however, needs to address this open question. Second, these results show that attentional mechanisms contribute to reappraisal and emotional experiences, but we did not assess clinical outcomes. An extended multiple session variant of the attentional control training may need to examine the long-term endurance of the effects observed in the current study as well as to test its effectiveness in improving depressive symptom severity and quality of life. Finally, the large individual differences in attentional control acquisition and the transfer of training showed that a subset of individuals took advantage of the training. Future research efforts need to identify (cognitive) markers to preselect individuals who may benefit from attentional control training procedures. 
In this study, attentional control training modified emotional attention bias, which in turn was associated with reappraisal and a reduction in experienced negative emotions. Our study provides clear evidence on the link between attentional mechanisms and reappraisal. Moreover, our new training provides an important step to personalized cognitive training of attention. 


\section{Acknowledgements}

This research was supported by a grant of the Research Foundation Flanders (FWO, reference 117438) awarded to Alvaro Sanchez and a grant BOF10/GOA/014 for a Concerted Research Action of Ghent University awarded to Ernst Koster. We are very grateful to Igor Marchetti and Megan T. deBettencourt for their assistance and comments on earlier versions of the manuscript. We also thank Jeroen Barbé, Naomi De Borger, and Ena Coenen for their help in the data collection. 


\section{References}

Aldao, A., Nolen-Hoeksema, S., \& Schweizer, S. (2010). Emotion-regulation strategies across psychopathology: A meta-analytic review. Clinical Psychology Review, 30(2), 217-237. http://doi.org/10.1016/j.cpr.2009.11.004

Augustine, A. a., \& Hemenover, S. H. (2009). On the relative effectiveness of affect regulation strategies: A meta-analysis. Cognition \& Emotion, 23(6), 1181-1220.

http://doi.org/10.1080/02699930802396556

Baert, S., De Raedt, R., \& Koster, E. (2010). Depression-related attentional bias: The influence of symptom severity and symptom specificity. Cognition \& Emotion, 24(6), 1044-1052. http://doi.org/10.1080/02699930903043461

Beard, C., Sawyer, A. T., \& Hofmann, S. G. (2012). Efficacy of attention bias modification using threat and appetitive stimuli: A meta-analytic review. Behavior Therapy, 43, 724-740. http://doi.org/10.1016/j.beth.2012.01.002

Bebko, G. M., Franconeri, S. L., Ochsner, K. N., \& Chiao, J. Y. (2011). Look before you regulate: Differential perceptual strategies underlying expressive suppression and cognitive reappraisal. Emotion (Washington, D.C.), 11(4), 732-742. http://doi.org/10.1037/a0024009

Bebko, G. M., Franconeri, S. L., Ochsner, K. N., \& Chiao, J. Y. (2014). Attentional Deployment Is Not Necessary for Successful Emotion Regulation via Cognitive Reappraisal or Expressive Suppression. Emotion (Washington, D.C.), 14(3), 504-512. http://doi.org/10.1037/a0035459

Beck, A. T., \& Haigh, E. A. P. (2014). Advances in cognitive theory and therapy: The generic cognitive model. Annual Review of Clinical Psychology, 10, 1-24. http://doi.org/10.1146/annurev-clinpsy-032813-153734

Beck, A. T., Steer, R. A., \& Brown, G. K. (1996). Manual for the Beck Depression Inventory II. San Antonio, TX: Psychological Corporation.

Bernstein, A., \& Zvielli, A. (2014). Attention Feedback Awareness and Control Training (AFACT): Experimental test of a novel intervention paradigm targeting attentional bias. Behaviour Research and Therapy, 55(1), 18-26. http://doi.org/10.1016/j.brat.2014.01.003

Clark, L. A., \& Watson, D. (1991). Tripartite model of anxiety and depression: psychometric evidence and taxonomic implications. Journal of Abnormal Psychology, 100(3), 316-36.

Clarke, P. J. F., Chen, N. T. M., \& Guastella, A. J. (2012). Prepared for the best: Readiness to modify attentional processing and reduction in anxiety vulnerability in response to therapy. Emotion, 12(3), 487-494. http://doi.org/10.1037/a0025592 
Clarke, P., Macleod, C., \& Shirazee, N. (2008). Prepared for the worst: readiness to acquire threat bias and susceptibility to elevate trait anxiety. Emotion (Washington, D.C.), 8(1), 4757. http://doi.org/10.1037/1528-3542.8.1.47

De Raedt, R., \& Koster, E. H. W. (2010). Understanding vulnerability for depression from a cognitive neuroscience perspective: A reappraisal of attentional factors and a new conceptual framework. Cognitive, Affective \& Behavioral Neuroscience, 10(1), 50-70. http://doi.org/10.3758/CABN.10.1.50

Duyck, W., Desmet, T., Verbeke, L. P. C., \& Brysbaert, M. (2004). WordGen: A tool for word selection and nonword generation in Dutch, English, German, and French. Behavior Research Methods, Instruments, \& Computers, 36(3), 488-499. http://doi.org/10.3758/BF03195595

Everaert, J., Duyck, W., \& Koster, E. H. W. (2014). Attention, interpretation, and memory biases in subclinical depression: A proof-of-principle test of the combined cognitive biases hypothesis. Emotion, 14(2), 331-340. http://doi.org/10.1037/a0035250

Everaert, J., Mogoase, C., David, D., \& Koster, E. H. W. (2014). Attention bias modification via single-session dot-probe training: Failures to replicate. Journal of Behavior Therapy and Experimental Psychiatry, 1-8. http://doi.org/10.1016/j.jbtep.2014.10.011

Gross, J. J. (2014). Emotion Regulation: Conceptual and Empirical Foundations. In J. J. Gross (Ed.), Handbook of emotion regulation. New York, NY: The Guilford Press.

Gross, J. J., \& John, O. P. (2003). Individual differences in two emotion regulation processes: implications for affect, relationships, and well-being. Journal of Personality and Social Psychology, 85(2), 348-362.

Hakamata, Y., Lissek, S., Bar-Haim, Y., Britton, J. C., Fox, N. a., Leibenluft, E., ... Pine, D. S. (2010). Attention bias modification treatment: A meta-analysis toward the establishment of novel treatment for anxiety. Biological Psychiatry, 68(11), 982-990.

http://doi.org/10.1016/j.biopsych.2010.07.021

Jamieson, J. P., Nock, M. K., \& Mendes, W. B. (2012). Mind over matter: Reappraising arousal improves cardiovascular and cognitive responses to stress. Journal of Experimental Psychology: General General, 141(3), 417-22. http://doi.org/10.1037/a0025719

Johnstone, T., van Reekum, C. M., Urry, H. L., Kalin, N. H., \& Davidson, R. J. (2007). Failure to regulate: counterproductive recruitment of top-down prefrontal-subcortical circuitry in major depression. The Journal of Neuroscience : The Official Journal of the Society for Neuroscience, 27(33), 8877-84. http://doi.org/10.1523/JNEUROSCI.2063-07.2007

Joormann, J., \& D'Avanzato, C. (2010). Emotion regulation in depression: Examining the role of cognitive processes. Cognition \& Emotion, 24(6), 913-939.

http://doi.org/10.1080/02699931003784939 
Lang, P. J., Bradley, M. M., \& Cuthbert, B. N. (2008). International affective picture system (IAPS): Affective ratings of pictures and instruction manual. Gainesville, FL: University of Florida.

MacLeod, C., Mathews, A., \& Tata, P. (1986). Attentional bias in emotional disorders. Journal of Abnormal Psychology, 95(1), 15-20. http://doi.org/10.1037/0021-843X.95.1.15

MacLeod, C., Rutherford, E., Campbell, L., Ebsworthy, G., \& Holker, L. (2002). Selective attention and emotional vulnerability: assessing the causal basis of their association through the experimental manipulation of attentional bias. Journal of Abnormal Psychology, 111(1), 107-123. http://doi.org/10.1037/0021-843X.111.1.107

Manera, V., Samson, A. C., Pehrs, C., Lee, I. A., \& Gross, J. J. (2014). The eyes have it: The role of attention in cognitive reappraisal of social stimuli. Emotion, 14(5), 833-839. http://doi.org/10.1037/a0037350

Mogoașe, C., David, D., \& Koster, E. H. W. (2014). Clinical efficacy of attentional bias modification procedures: An updated meta-analysis. Journal of Clinical Psychology, 70(12), 1133-1157. http://doi.org/10.1002/jclp.22081

Morris, J. a, Leclerc, C. M., \& Kensinger, E. a. (2014). Effects of valence and divided attention on cognitive reappraisal processes. Social Cognitive and Affective Neuroscience, 1-10. http://doi.org/10.1093/scan/nsu004

Ochsner, K. N., Bunge, S. a, Gross, J. J., \& Gabrieli, J. D. E. (2002). Rethinking feelings: an FMRI study of the cognitive regulation of emotion. Journal of Cognitive Neuroscience, 14(8), 1215-1229. http://doi.org/10.1162/089892902760807212

Preacher, K. J., \& Hayes, A. F. (2008). Asymptotic and resampling strategies for assessing and comparing indirect effects in multiple mediator models. Behavior Research Methods, 40(3), 879-91. http://doi.org/10.3758/BRM.40.3.879

Preacher, K. J., \& Kelley, K. (2011). Effect size measures for mediation models: quantitative strategies for communicating indirect effects. Psychological Methods, 16(2), 93-115. http://doi.org/10.1037/a0022658

Sanchez, A., Everaert, J., De Putter, L., Mueller, S., \& Koster, E. H. W. (2015). Life is ... great! Emotional attention during instructed and uninstructed ambiguity resolution in relation to depressive symptoms. Biological Psychology, 109, 67-72. http://doi.org/10.1016/j.biopsycho.2015.04.007

Schnyer, D. M., Beevers, C. G., deBettencourt, M. T., Sherman, S. M., Cohen, J. D., Norman, K. a, \& Turk-Browne, N. B. (2015). Neurocognitive therapeutics: from concept to application in the treatment of negative attention bias. Biology of Mood \& Anxiety Disorders, 5(1), 1619. http://doi.org/10.1186/s13587-015-0016-y 
Segal, Z. V, Kennedy, S., Gemar, M., Hood, K., Pedersen, R., \& Buis, T. (2006). Cognitive reactivity to sad mood provocation and the prediction of depressive relapse. Archives of General Psychiatry, 63(7), 749-755. http://doi.org/10.1001/archpsyc.63.7.749

Sheppes, G., Suri, G., \& Gross, J. J. (2015). Emotion Regulation and Psychopathology. Annual Review of Clinical Psychology, 11, 379-405. http://doi.org/10.1146/annurev-clinpsy032814-112739

Urry, H. L. (2010). Seeing, thinking, and feeling: emotion-regulating effects of gaze-directed cognitive reappraisal. Emotion (Washington, D.C.), 10(1), 125-135. http://doi.org/10.1037/a0017434

Van der Does, A. J. W. (2002). Handleiding bij de Nederlandse versie van de Beck Depression Inventory - second edition (BDI-II-NL). [The Dutch version of the Beck Depresion Inventory-II]. Lisse, The Netherlands: Swets \& Zeitlinger.

Van Reekum, C. M., Johnstone, T., Urry, H. L., Thurow, M. E., Schaefer, H. S., Alexander, A. L., \& Davidson, R. J. (2007). Gaze fixations predict brain activation during the voluntary regulation of picture-induced negative affect. NeuroImage, 36(3), 1041-1055. http://doi.org/10.1016/j.neuroimage.2007.03.052

Vanderhasselt, M. A., Kühn, S., \& De Raedt, R. (2013). "Put on your poker face": Neural systems supporting the anticipation for expressive suppression and cognitive reappraisal. Social Cognitive and Affective Neuroscience, 8(8), 903-910. http://doi.org/10.1093/scan/nss090

Webb, T. L., Miles, E., \& Sheeran, P. (2012). Dealing with feeling: A meta-analysis of the effectiveness of strategies derived from the process model of emotion regulation. Psychological Bulletin, 138(4), 775-808. http://doi.org/10.1037/a0027600

Watson, D., Clark, L. A., Weber, K., Assenheimer, J. S., Strauss, M. E., \& McCormick R. A. (1995) Testing a tripartite model: I. Evaluating the convergent and discriminant validity of anxiety and depression symptom scales. Journal of Abnormal Psychology, 104, 3-14. http://doi.org/10.1037/0021-843X.104.1.3 
Table 1. Sample characteristics and descriptive statistics.

\begin{tabular}{|c|c|c|c|c|}
\hline \multirow[b]{2}{*}{ Variables } & \multicolumn{2}{|c|}{ Training $(N=18)$} & \multicolumn{2}{|c|}{ Control $(N=18)$} \\
\hline & $M$ & $S D$ & $M$ & $S D$ \\
\hline Gender (male/female) & \multicolumn{2}{|c|}{$3 / 15$} & \multicolumn{2}{|c|}{$3 / 15$} \\
\hline Age & 21.06 & 3.26 & 21.35 & 4.77 \\
\hline BDI-II & 14.78 & 10.61 & 10.35 & 7.38 \\
\hline \multicolumn{5}{|l|}{ Attentional control training indices } \\
\hline Interpretation bias baseline phase (prop) & 0.72 & 0.24 & 0.74 & 0.20 \\
\hline Interpretation bias modification phase (prop) & 0.99 & 0.01 & 0.75 & 0.17 \\
\hline Attention bias baseline phase (prop) & 0.50 & 0.04 & 0.52 & 0.04 \\
\hline Attention bias modification phase (prop) & 0.63 & 0.08 & 0.50 & 0.03 \\
\hline \multicolumn{5}{|l|}{ Pre-training indices } \\
\hline Dot-probe RT congruent trials (ms) & 525.34 & 101.07 & 479.43 & 62.11 \\
\hline Dot-probe RT incongruent trials (ms) & 522.61 & 112.97 & 479.75 & 67.03 \\
\hline Dot-probe attention bias (d score in $\mathrm{ms}$ ) & -2.72 & 34.07 & 0.32 & 16.28 \\
\hline Reappraisal (range 0-4) & 2.10 & 0.71 & 1.90 & 0.77 \\
\hline Negative emotion after appraisal (range 0-9) & 5.92 & 1.56 & 5.90 & 1.75 \\
\hline Negative emotion after reappraisal (range $0-9$ ) & 4.62 & 1.24 & 4.85 & 1.76 \\
\hline \multicolumn{5}{|l|}{ Post-training indices } \\
\hline Dot-probe RT congruent trials (ms) & 506.52 & 116.53 & 437.71 & 53.14 \\
\hline Dot-probe RT incongruent trials (ms) & 494.12 & 126.40 & 436.63 & 52.61 \\
\hline Dot-probe attention bias (d score in $\mathrm{ms}$ ) & -12.39 & 43.94 & -1.08 & 17.88 \\
\hline Reappraisal (range 0-4) & 2.11 & 0.75 & 1.97 & 0.77 \\
\hline Negative emotion after appraisal (range 0-9) & 5.51 & 1.84 & 5.46 & 2.08 \\
\hline Negative emotion after reappraisal (range $0-9$ ) & 4.74 & 1.66 & 4.64 & 1.74 \\
\hline
\end{tabular}

Notes. $\mathrm{M}=$ Mean; $\mathrm{SD}=$ Standard deviation; $\mathrm{RT}=$ reaction time; $\mathrm{ms}=$ millisecond prop = proportion 
Table 2. Correlations between the training condition and change scores.

\begin{tabular}{lccccc}
\hline Variables & 1 & 2 & 3 & 4 & 5 \\
\hline $\begin{array}{l}\text { 1. Training Condition } \\
\text { (0- Control; 1 - Training) }\end{array}$ & - & $.70^{* *}$ & -.14 & .03 & .11 \\
2. Attentional control acquisition (training) & - & $-.45^{* *}$ & .02 & .04 \\
3. Attention bias change (dot-probe) & & - & $-.39^{*}$ & .08 \\
4. Reappraisal change & & & - & $-.45^{* *}$ \\
5. Negative Emotion after Reappraisal change & & & & & -
\end{tabular}

Note. ${ }^{+} p<.10 ; * p<.05 ; * * p<.01$ 
Table 3. Indirect effect models tested with condition as predictor, reappraisal change as outcome, and attentional control acquisition and attention bias change as potential mediators

\begin{tabular}{ccccc}
\hline $\begin{array}{c}\text { Indirect Effect } \\
\text { Model }\end{array}$ & Effect $(a \times b)$ & SE & CI (lower) & CI (upper) \\
\hline Total & -.3376 & .3653 & -1.1590 & .2418 \\
1 (Alternative) & -.4821 & .3502 & -1.2867 & .0621 \\
$\mathbf{2}$ (Hypothesized) & $\mathbf{. 4 7 4 7}$ & $\mathbf{. 4 0 6 0}$ & $\mathbf{. 0 0 6 1}$ & $\mathbf{1 . 6 2 3 8}$ \\
3 (Alternative) & -.3302 & .3349 & -1.3122 & .0509 \\
\hline
\end{tabular}

Indirect Effect Models:

1: Condition $\rightarrow$ Attentional control acquisition $\rightarrow$ Reappraisal change; $n . s$.

2: Condition $\rightarrow$ Attentional control acquisition $\rightarrow$ Attention bias change $\rightarrow$ Reappraisal change; $p<.05$

3: Condition $\rightarrow$ Attention bias change $\rightarrow$ Reappraisal change; $n . s$.

Notes: SE $=$ Standard error; CI (lower) $=$ lower bound of a 95\% confidence interval; CI (upper) $=$ upper bound; $\rightarrow=$ affects; n.s. $=$ non-significant 
Table 4. Indirect effect models tested with Condition as predictor, Negative Emotions after Reappraisal change as outcome, and Attentional Control Acquisition, Attention bias change and Reappraisal change as potential mediators

\begin{tabular}{ccccc}
\hline $\begin{array}{c}\text { Indirect Effect } \\
\text { Model }\end{array}$ & Effect $(a \times b)$ & Boot SE & Boot LLCI & Boot ULCI \\
\hline Total & -.2045 & .4740 & -1.3209 & .4598 \\
1 (Alternative) & -.2189 & .4671 & -1.6448 & .3342 \\
2 (Alternative) & .1394 & .3022 & -.1305 & 1.3130 \\
3 (Alternative) & .2276 & .1768 & -.0066 & .7078 \\
$\mathbf{4}$ (Hypothesized) & -.2241 & .2247 & -.9512 & -.0045 \\
5 (Alternative) & -.0970 & .2491 & -1.1755 & .0879 \\
6 (Alternative) & .1559 & .1860 & -.0188 & 7989 \\
7 (Alternative) & -.1875 & .2420 & -.7696 & .1492 \\
\hline
\end{tabular}

Indirect Effect Models:

1: Condition $\rightarrow$ Attentional control acquisition $\rightarrow$ Negative emotions change; $n$.s.

2: Condition $\rightarrow$ Attentional control acquisition $\rightarrow$ Attention bias change $\rightarrow$ Negative emotions change; n.s.

3: Condition $\rightarrow$ Attentional control acquisition $\rightarrow$ Reappraisal change $\rightarrow$ Negative emotions change; n.s.

4: Condition $\rightarrow$ Attentional control acquisition $\rightarrow$ Attention bias change $\rightarrow$ Reappraisal change $\rightarrow$ Negative emotions change; $p<.05$

5: Condition $\rightarrow$ Attention bias change $\rightarrow$ Negative emotions change; $n$.s.

6: Condition $\rightarrow$ Attention bias change $\rightarrow$ Reappraisal change $\rightarrow$ Negative emotions change; n.s.

7: Condition $\rightarrow$ Reappraisal change $\rightarrow$ Negative emotions change; $n . s$.

Notes: $\mathrm{SE}=$ Standard error; CI (lower) $=$ lower bound of a 95\% confidence interval; CI (upper) $=$ upper bound; $\rightarrow=$ affects; n.s. $=$ non significant 
Figure 1. Schematic on the task sequence during the experimental session, and overview of indices computed in each task

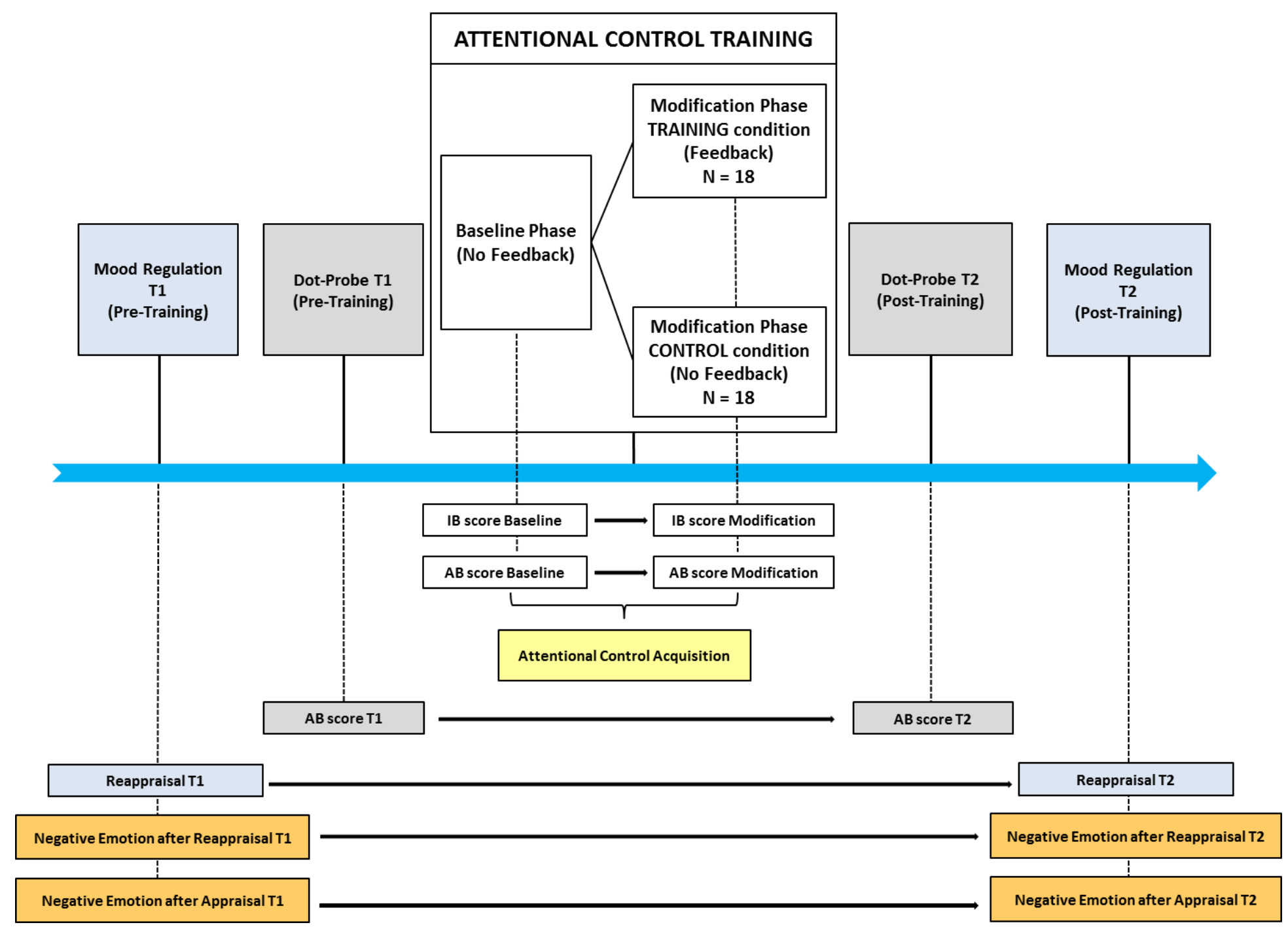

Notes. $\mathrm{IB}=$ Interpretation bias; $\mathrm{AB}=$ Attention bias; $\mathrm{T} 1=$ Time 1 (pre-training); $\mathrm{T} 2=$ Time 2 (post-training) 
Figure 2. Schematic on the basic trial sequence in the SST combined with ET.

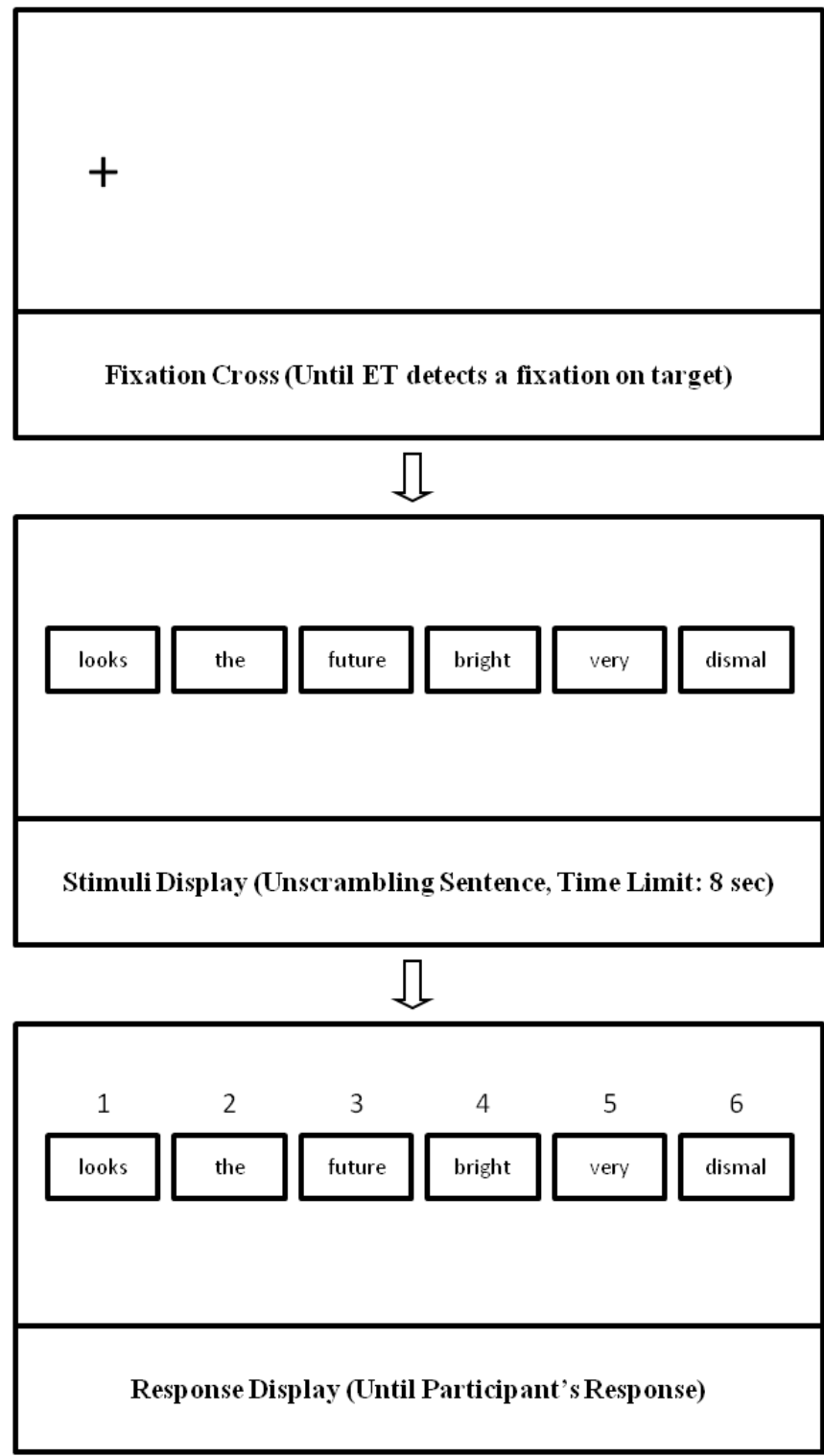

Notes. SST $=$ Scramble Sentence Task $;$ ET $=$ Eye-tracker; sec $=$ seconds 
Figure 3. Schematic overview of the attentional control training procedure

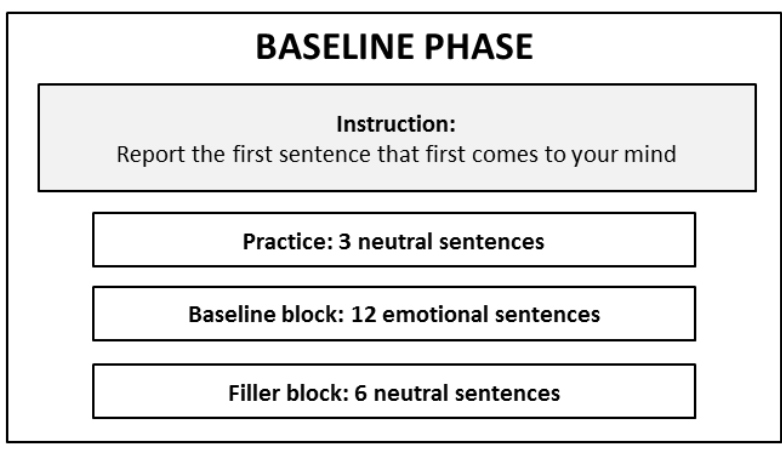

\begin{tabular}{|c|c|c|}
\hline Control Condition \\
Instruction: \\
Report the first sentence that first comes to your mind \\
No Feedback
\end{tabular}


Figure 4. Flow of trial events in the dot-probe task

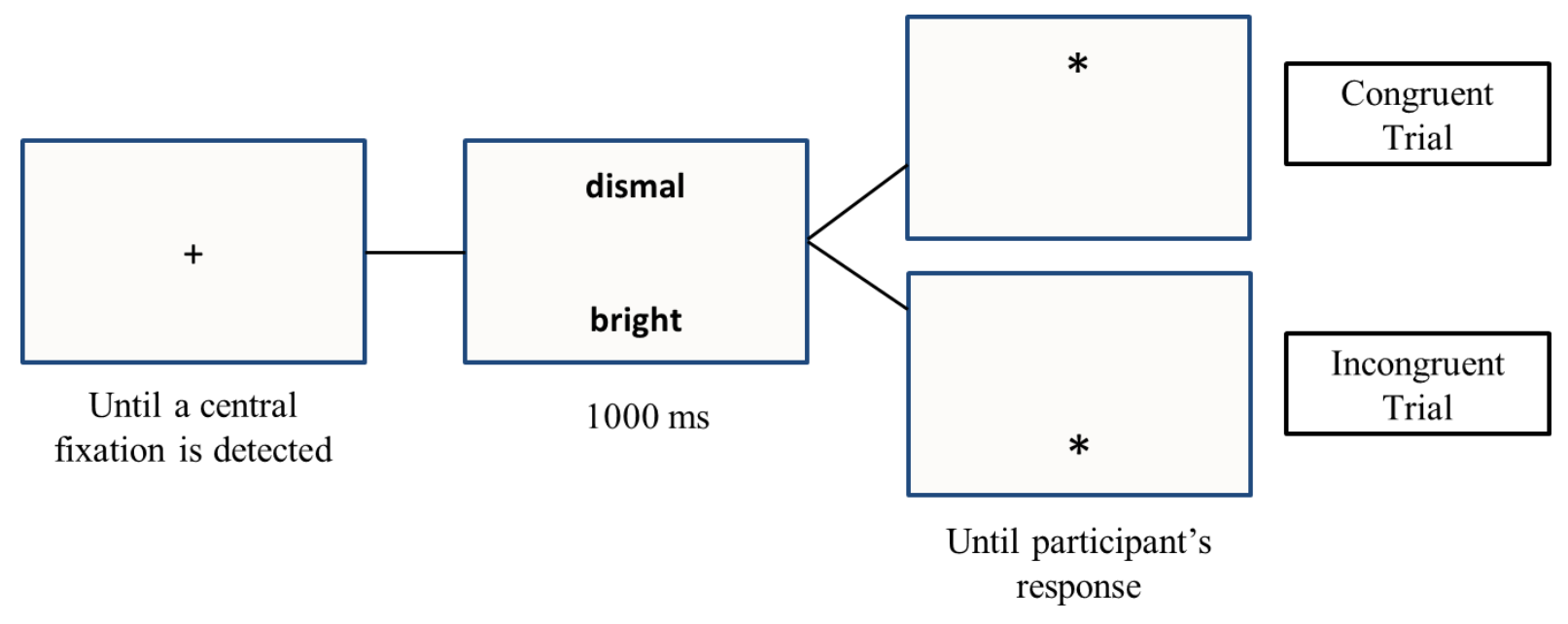

$\mathrm{AB}$ score $=\mathrm{RTs}$ Incongruent Trials $-\mathrm{RTs}$ Congruent Trials (larger scores $=$ larger negative $\mathrm{AB}$ )

Notes. $\mathrm{AB}=$ Attention bias; $\mathrm{RT}=$ Reaction time 
Figure 5. Flow of trial events in the emotion regulation task.
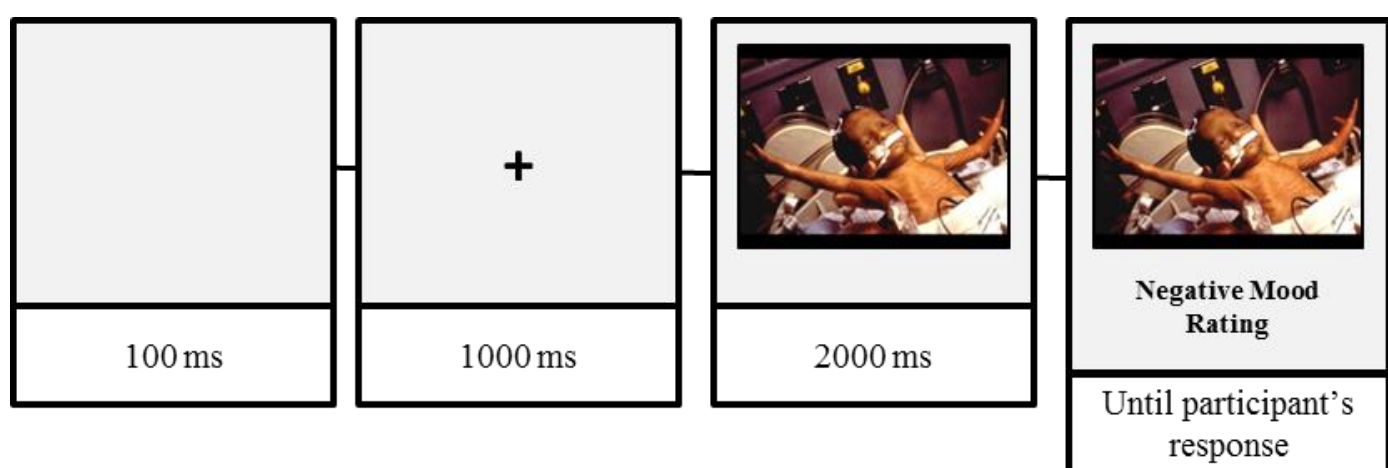
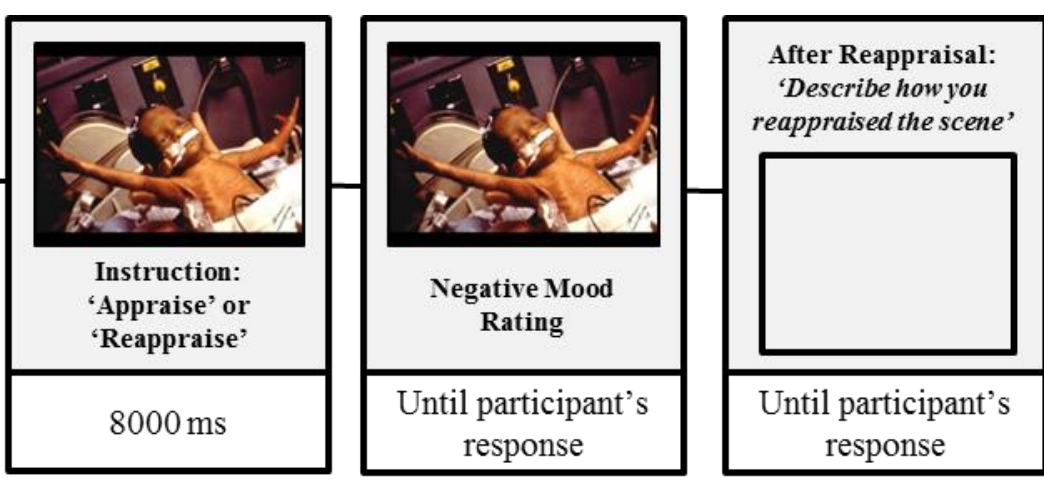

Note: $\mathrm{ms}=$ milisesonds 
Figure 6. Relationship among attention control acquisition in the training and attention bias change levels in the dot-probe at each modification condition.

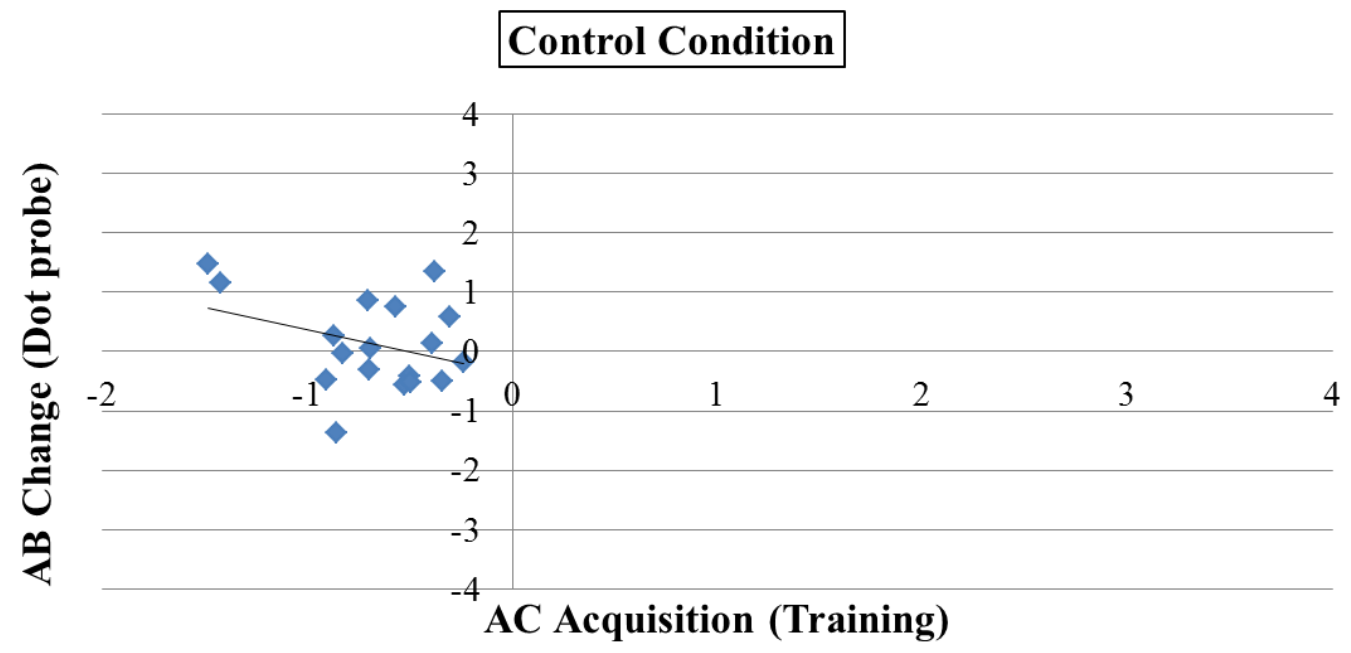

\section{Training Condition}

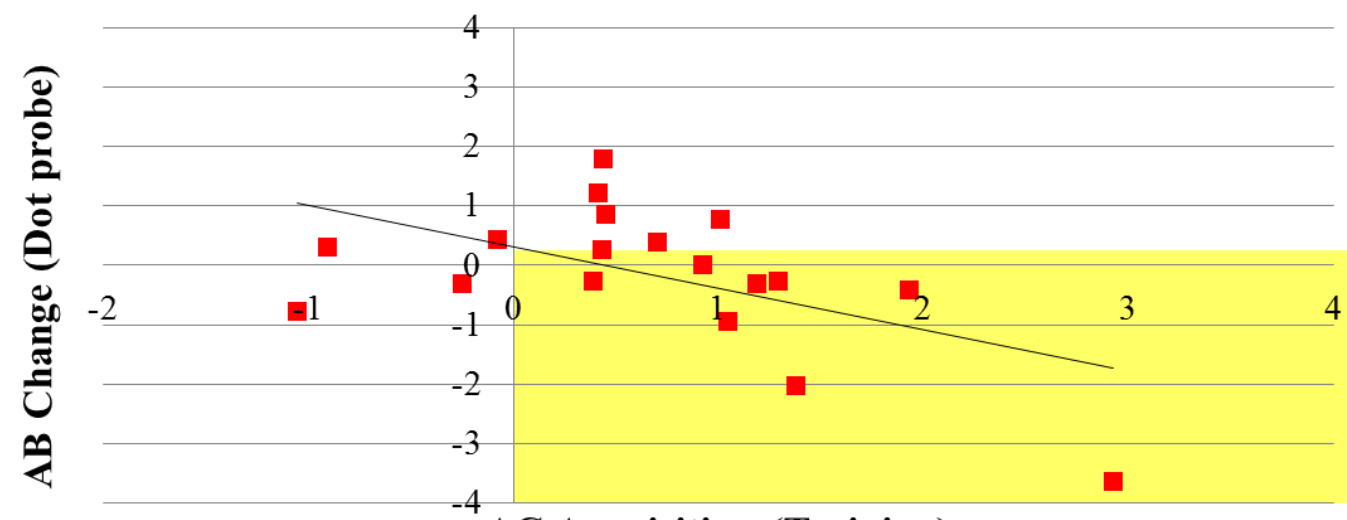

AC Acquisition (Training)

Notes. $\mathrm{AC}=$ attention control (training); $\mathrm{AB}=$ attention bias (dot-probe)

$\mathrm{AC}$ acquisition and $\mathrm{AB}$ change are measured by standardized residuals (positive scores $=$ increases from Time 1 to Time 2; negative scores $=$ decreases from $\mathrm{T} 1$ to $\mathrm{T} 2$ )

In the control condition, neither $\mathrm{AC}$ acquisition in the training nor $\mathrm{AB}$ changes in the dot probe task were observed. In the training condition, individual differences in $\mathrm{AC}$ acquisition in the training predicted the level of $\mathrm{AB}$ change in the dot probe task. Specific dispersion area on the association between $\mathrm{AC}$ acquisition increases and $\mathrm{AB}$ decreases in the Training condition is highlighted. The highlighted dispersion area indicates that only the participants in the Training condition who showed the largest $\mathrm{AC}$ acquisition were the ones who also showed a reduction in the negative $\mathrm{AB}$. 
Figure 7. Overview of the indirect effect model supported: Attention training leads to increases in $\mathrm{AC}$ acquisition (in the training); larger $\mathrm{AC}$ acquisition leads to larger $\mathrm{AB}$ reduction (in the dotprobe); larger $\mathrm{AB}$ reduction leads to larger reappraisal increases; larger reappraisal increases leads to larger reductions in negative emotion after reappraisal

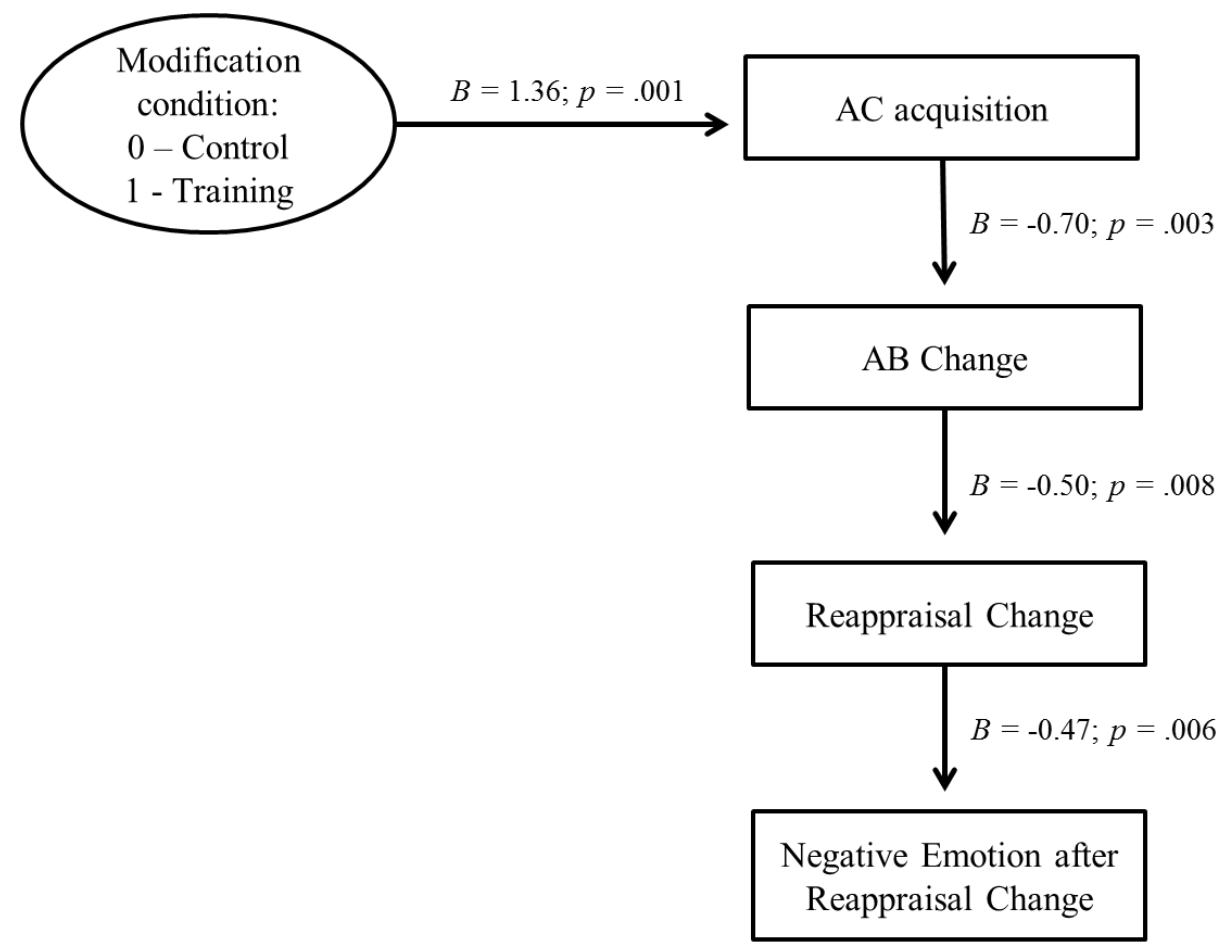

Indirect effect model $(1.36 \times-0.70 \times-0.50 \times-0.47)=-0.22 ; \mathrm{SE}=0.22 ; 95 \%-\mathrm{CI}:-.9512,-.0045$

Notes. $\mathrm{AC}=$ attention control (training); $\mathrm{AB}=$ attention bias (dot-probe)

$\mathrm{B}=\mathrm{Beta} ; \mathrm{SE}=$ Standardized error $\mathrm{CI}=$ Confidence Interval 